\title{
Constraint effects on the elastic-plastic fracture behaviour in strain gradient solids
}

\author{
Y. WEI \\ LNM, Institute of Mechanics, Chinese Academy of Sciences, Beijing 100080, China \\ Received in final form 14 August 2001
}

\begin{abstract}
A B S T R A C T Crack-tip constraint effects (or T-stress effects) on the elastic-plastic fracture behaviour in strain gradient materials are analysed in the present study. The T-stress effects on the stress distributions along the plane ahead of the stationary and growing crack tip, respectively, are analysed by using the Fleck and Hutchinson strain gradient plasticity formation. For a steadily growing crack, the T-stress effects on the steady-state fracture toughness are analysed by adopting the embedded fracture process zone model. In addition, the analysis for the growing crack is applied to an interfacial cracking experiment for a metal/ceramic system, and the material length-scale parameter appearing in the strain gradient plasticity theory is predicted. In the present analyses, a new finite element method specially designed for strain gradient problems by Wei and Hutchinson is adopted.
\end{abstract}

Keywords fracture toughness; metal/ceramic interface; strain gradient plasticity; T-stress.

\section{INTRODUCTION}

For conventional elastic-plastic material, the effect of crack-tip constraint on fracture behaviour of ductile materials has attracted a great deal of interest in the past 2 decades. Starting from checking the elastic-plastic crack tip fields, for specimens with different crack geometries by using the finite element method, Larsson and Carlsson $^{1}$ first presented the role of the nonsingular stress acting parallel to the crack surface, Rice $^{2}$ gave the analytical interpretation of this nonsingular stress effect, and discussed the influence on plastic zone size in detail. Based on small scale yielding conditions, $\mathrm{Li}$ and $\mathrm{Wang}^{3}$ investigated the constraint effect on the elastic-plastic fracture behaviour from the second term of the elasticplastic asymptotic solution. Betegon and Hancock, ${ }^{4}$ and Du and Hancock ${ }^{5}$ have studied the effect of the nonsingular stress term $\mathrm{T}$ (or $\mathrm{T}$-stress) in elastic asymptotic solutions, acting parallel to the crack plane, on elasticplastic crack-tip fields. These authors found that the

Correspondence: Y. Wei, LNM, Institute of Mechanics, Chinese Academy of Sciences, Beijing 100080, China. E-mail: Ywei@Lnm.imech.ac.cn second term of the asymptotic solution for either elastic fracture or elastic-plastic fracture had a strong influence on the crack tip fields. For the constraint effects on the elastic-plastic fracture behaviour, based on the asymptotic expressions, O'Dowd and Shih ${ }^{6,7}$ have developed a J-Q two-parameter fracture criterion, while Wei and Wang ${ }^{8,9}$ have developed a J-Q-k2 revised two-parameter criterion, where $\mathrm{Q}$ and $\mathrm{k} 2$ characterize the constraint effects. The two- or revised two-parameter criteria mentioned above have effectively described the crack tip field from small scale yielding to large scale yielding and from bending-dominated fracture to lower-triaxiality stress-dominated fracture. Alternatively, Tvergaard and Hutchinson ${ }^{10-12}$ have studied the behaviour of growing cracks, the fracture work, and constraint effects as well by using an embedded process zone model (EPZ Model; or cohesive zone model) presented by Needleman. ${ }^{13}$ They also adopted another two-parameter criterion based on the parameters $\left(\Gamma_{0}, \hat{\sigma}\right)$, where $\Gamma_{0}$ and $\hat{\sigma}$ are the fracture work for advancing the crack surface per unit area and the maximum separation strength, respectively. The cohesive zone model has attracted a great deal of interest recently in the elastic-plastic fracture research area. This 
is because the dominant parameters, the fracture work per unit area and the maximum separation strength, not only have direct physical meanings, but can be readily connected and measured in fracture experiments. ${ }^{14-16}$

An important effect recently observed in fracture experiments is that a strong size effect on fracture at the microscale along a metal or an interface of a metal/ceramic system causes considerable elevation of the maximum separation stress during crack growth. However, the phenomenon cannot be predicted by the conventional elastic-plastic theory. Size effects have also been found in other kinds of experiments, such as microindentation tests ${ }^{17-20}$ and torsion tests on thin copper wires ${ }^{21}$ as well as bending tests of thin beams. ${ }^{22}$ In order to interpret and predict the size effects, several versions of strain gradient plasticity theories have recently been presented, ${ }^{23-27}$ which have been formed basically through introducing some length-scale parameters in the constitutive equations. Using the length-scale parameters, the size effects can be characterized and further predicted. Some applications of these strain gradient plasticity theories have been made, such as simulations for the microindentation tests ${ }^{20,28-30}$ and for stationary and growing crack tip fields and the application to thin film delamination. ${ }^{31-34}$ In particular, for steadily growing crack-tip fields under small scale yielding, the maximum separation stress near the crack tip can reach very high values, up to 10 times the material yield stress, according to strain gradient plasticity theory. ${ }^{32}$ This result may be applicable to some extent to further efforts in exploring the linkage between macroscopic cracking and atomistic fracture. ${ }^{35}$

For any particular fracture problem (a practical fracture structure geometry, as shown in Fig. 1a), constraint effects are usually inevitable. This poses the problem of finding the elastic-plastic fracture behaviour for a strain gradient solid given the level of imposed constraint. This paper deals with this through a detailed analysis of constraint effects on the fracture of elastic-plastic strain gradient material. The analyses include not only the stationary crack case, but also the growing crack case. The T-stress effects on the stress distributions along the plane ahead of the crack tip are analysed by using the Fleck and Hutchinson ${ }^{24}$ strain gradient plasticity theory. In addition, for the steadily growing crack, the T-stress effects on the fracture toughness are analysed by using the EPZ model. Furthermore, the analysis for the growing crack case is applied to an interfacial cracking experiment for a metal/ceramic system, ${ }^{16}$ and the material length parameters included in the strain gradient theory are predicted. In the present analysis, a new finite element method, specially designed for strain gradient problems ${ }^{36}$ is adopted. (a)

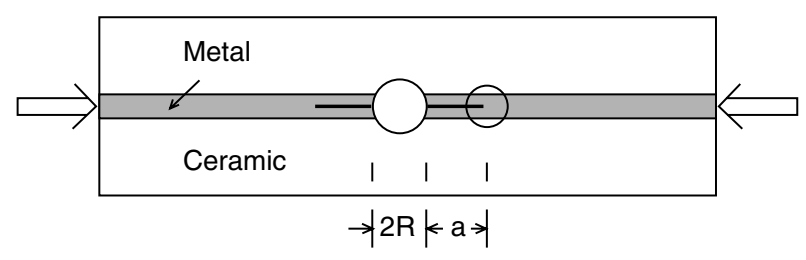

(b)

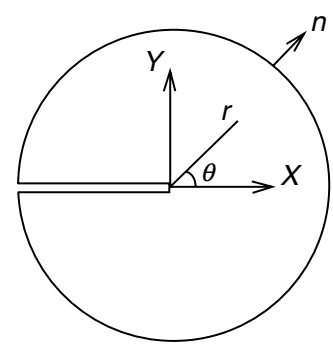

Fig. 1 A growing crack specimen (a), and a simplified analytical model subjected to the $\mathrm{K}$ field including the nonsingular T-stress on the boundary (b).

\section{PROBLEM FORMULATION}

For a practical crack geometry (or an experimental fracture specimen, as shown in Fig. 1a) under loading, the loading process towards fracture can be equivalently described by a simplified problem, a typical K-field augmented with the effects of the T-stress, as shown in Fig. 1b. The T-stress is the second term in the elastic asymptotic field (a nonsingular term)

$\sigma_{i j}=\frac{K}{\sqrt{2 \pi r}} \bar{\sigma}_{i j}(\theta)+T \delta_{1 i} \delta_{1 j}$

where $(r, \theta)$ are polar coordinates and $\delta_{i j}$ is the Kronecker delta. The $\bar{\sigma}_{i j}(\theta)$ is the angular distributed function available in any fracture textbook. For Mode I loading, $K$ is the amplitude of the singular field. For a stationary crack or for a growing crack, the stress-strain field near the crack tip can be roughly divided into three subfields. One field is an elastic field far away from the crack tip. The second field is a strain-gradient dominated field, which surrounds and is very close to the tip. The third field is the conventional elastic-plastic field located in the intermediate region between the elastic field and the strain gradient field. Whenever one can combine an evaluation of the local crack tip field with a material fracture criterion it becomes possible to assess the material fracture properties and evaluate the structural implications. In the present research work, our attention is focused on exploring the T-stress effects on the elastic-plastic fracture behaviour in a strain gradient solid for stationary and steadily growing cracks. 


\section{Deformation theory of strain gradient plasticity}

For a stationary crack, the deformation theory of Fleck and Hutchinson strain gradient plasticity ${ }^{23,24}$ will be adopted. The general expression for this theory for a compressible solid has been derived by Wei et al. ${ }^{20}$ Here a brief description of the result is given.

The constitutive relations can be expressed as

$$
\begin{gathered}
\sigma_{i j}=\frac{E}{1+v+\frac{3}{2} E / h^{p}} \varepsilon_{i j}+\frac{1}{3}\left(\frac{E}{1-2 v}-\frac{E}{1+v+\frac{3}{2} E / h^{p}}\right) \varepsilon_{k k} \delta_{i j} \\
\tau_{i j k}=2 E\left\{\sum_{I=1}^{3} \frac{L_{I}^{2}}{L_{I}^{2} / L_{I}^{e 2}+2 E / h^{p}} T_{i j k l m n}^{(I)}+L_{4}^{e 2} T_{i j k l m n}^{(4)}\right\} \eta_{l m n}(2)
\end{gathered}
$$

where $h^{p}$ is the equivalent plastic modulus. Its definition is

$h^{p}=\Sigma /(\Xi-\Sigma / E)$

where

$$
\begin{aligned}
& \Sigma=\sqrt{3 \mathcal{F}_{2}}=\sqrt{\frac{3}{2} \sigma_{i j}^{\prime} \sigma_{i j}^{\prime}+\sum_{I=1}^{3} L_{I}^{-2} \tau_{i j k}^{(I)} \tau_{i j k}^{(I)}} \\
& \Xi=\sqrt{\frac{2}{3} \varepsilon_{i j}^{\prime} \varepsilon_{i j}^{\prime}+\sum_{I=1}^{3} L_{I}^{2} \eta_{i j k}^{(I)} \eta_{i j k}^{(I)}}
\end{aligned}
$$

$\Sigma$ and $\Xi$ are the effective stress and effective strain, respectively. $L_{I}^{e}(I=1,4)$ and $L_{I}(I=1,3)$ are the elastic and plastic length-scale parameters, respectively. $L_{I}(I=1,3)$ characterize the strength of the energy density contributed from the deviatoric part of the strain gradient, and $L_{I}^{e}(\mathrm{I}=1,3)$ characterize the elastic part. $L_{4}^{e}$ characterizes the strength of the elastic energy density contributed from the hydrostatic part of the strain gradient. From the discussion of Fleck and Hutchinson, ${ }^{24}$ for the stretch and rotation gradient (SG solid) there is a relation between the plastic length parameters of the form

$L_{1}=L, \quad L_{2}=\frac{1}{2} L, \quad L_{3}=\sqrt{\frac{5}{24}} L$

Similarly, the form of Eq. (5) is also valid for the elastic length parameters by changing each $L$ into the correspond$\operatorname{ing} L^{e}$. Moreover, previous research work has shown that the solution of the strain gradient problem is insensitive to the value of $L^{e} / L$ within the region $0<L^{e} / L<1 .^{32}$ Thus we can, for convenience, take $L^{e} / L=0.5$ in the present research work. In addition, we take $L_{4}^{e}=L^{e} / 2$. In Eq. (2) $T_{i j k l m n}^{(I)}$ $(I=1,4)$ are the projection tensors of the strain gradients The detailed expressions for these were derived by Wei and Hutchinson; ${ }^{32}$ for ease of reference they are set out in the appendix of the present paper. $\tau_{i j k}$ and $\eta_{i j k}=u_{k, i j}$ are the higher-order stress and strain, respectively.
Furthermore, considering power-law strain hardening material,

$\Xi=\Xi_{0}\left(\Sigma / \sigma_{Y}\right), \quad \Sigma \leq \sigma_{Y} ; \quad \Xi=\Xi_{0}\left(\Sigma / \sigma_{Y}\right)^{1 / N}, \quad \Sigma>\sigma_{Y}$

one has the expression for the equivalent plastic modulus $h^{p}$ as

$h^{p}=E\left\{\left(\Sigma / \sigma_{Y}\right)^{1 / N-1}-1\right\}^{-1}$

\section{Flow theory of strain gradient plasticity}

The flow theory of Fleck and Hutchinson strain gradient plasticity must be used for a growing crack. The General expressions for this have been derived in Wei and Hutchinson. ${ }^{32}$ Here a brief description of the results are set out for convenient reference.

The constitutive relations are dictated as

$$
\begin{aligned}
\dot{\sigma}_{i j}= & {\left[L_{i j k l}-\frac{9 \mu}{(3+H) \sigma_{e}^{2}} \sigma_{i j}^{\prime} \sigma_{k l}^{\prime}\right] \dot{\varepsilon}_{k l} } \\
+ & {\left[-\frac{6 E\left(L^{e} / L\right)^{2}}{(3+H) \sigma_{e}^{2}} \sigma_{i j}^{\prime} \sum_{I=1}^{3}\left(\frac{k_{e}^{(I)}}{k^{(I)}}\right)^{2} \tau_{k l m}^{(I)}\right] \dot{\eta}_{k l m} } \\
\dot{\tau}_{i j k}= & {\left[-\frac{6 E\left(L^{e} / L\right)^{2}}{(3+H) \sigma_{e}^{2}} \sigma_{m n}^{\prime} \sum_{I=1}^{3}\left(\frac{k_{e}^{(I)}}{k^{(I)}}\right)^{2} \tau_{i j k}^{(I)}\right] \dot{\varepsilon}_{m n} } \\
+ & {\left[\mathcal{f}_{i j k l m n}-\frac{8(1+v) E\left(L^{e} / L\right)^{4}}{(3+H) \sigma_{e}^{2}} \sum_{I=1}^{3}\left(\frac{k_{e}^{(I)}}{k^{(I)}}\right)^{2} \tau_{i j k}^{(I)}\right.} \\
& \left.\sum_{\mathcal{f}=1}^{3}\left(\frac{k_{e}^{(f)}}{k^{(f)}}\right)^{2} \tau_{l m n}^{(\mathcal{I})}\right] \dot{\eta}_{l m n}
\end{aligned}
$$

where $\sigma_{e}=\sqrt{3 \sigma_{i j}^{\prime} \sigma_{i j}^{\prime} / .2}$ and $\mu=\mathrm{E} /[2(1+v)]$ are the conventional effective stress and shear modulus, respectively, and

$L_{i j k l}=\frac{E}{2(1+v)}\left[\delta_{i j} \delta_{j l}+\delta_{i l} \delta_{j k}+\frac{2 v}{(1-2 v)} \delta_{i j} \delta_{k l}\right]$

$\mathcal{f}_{i j k l m n}=2 E L^{e 2} \sum_{I=1}^{4} k_{e}^{(I) 2} T_{i j k l m n}^{(I)}$

$H=\left(\frac{\Sigma}{\sigma_{e}}\right)^{2}\left[1+\frac{2 E L^{e 2}}{b \Sigma^{2} L^{4}} \sum_{I=1}^{3} \frac{\left(k_{e}^{(I)}\right)^{2}}{\left(k^{(I)}\right)^{4}} \tau_{i j k}^{(I)} \tau_{i j k}^{(I)}\right] \frac{b}{\mu}$

where $k_{e}^{(I)}=L_{I}^{e} / L^{e}$ and $k^{(I)}=L_{I} / L(I=1,4), b$ is the plastic tangential modulus and its expression is

$b=E\left\{N^{-1}\left(\Sigma / \sigma_{Y}\right)^{1 / N-1}-1\right\}^{-1}$

For power-law strain hardening material, see (6). 


\section{Steady-state crack growth}

For a steadily growing crack problem, the rate-independent incremental constitutive Eq. (8) can be replaced by a full-quantity equation through considering the steadystate relations

$\left(\dot{\tau}_{i j k}, \dot{\eta}_{i j k}, \dot{\sigma}_{i j}, \dot{\varepsilon}_{i j}\right)=-\dot{a}\left(\frac{\partial \tau_{i j k}}{\partial x}, \frac{\partial \eta_{i j k}}{\partial x}, \frac{\partial \sigma_{i j}}{\partial x}, \frac{\partial \varepsilon_{i j}}{\partial x}\right)$

where $\dot{a}$ is the velocity of crack tip advance. Substituting Eq. (11) into Eq. (8), the equations, independent of the crack tip velocity, are obtained and can be expressed by the matrix form as

$\left\{\begin{array}{l}\frac{\partial \sigma}{\partial x} \\ \frac{\partial \tau}{\partial x}\end{array}\right\}=\left[\begin{array}{ll}A & B \\ B & C\end{array}\right]\left\{\begin{array}{l}\frac{\partial \varepsilon}{\partial x} \\ \frac{\partial \eta}{\partial x}\end{array}\right\}$

where the expressions for matrices $\mathrm{A}, \mathrm{B}$ and $\mathrm{C}$ can be readily obtained from the Eq. (8) and are not shown here.

A special finite element method based on the above mentioned features of the steadily growing crack problem has been presented by Dean and Hutchinson. ${ }^{37}$ Using such a finite element method, one can directly obtain a full-quantity solution through iterating to solve the finite element equations with the full-quantity variables, instead of the increment ones.

\section{Stress distributions along the plane ahead of the crack tip}

A marked characteristic of strain gradient effects in a fracture problem is that the stress distribution along the plane ahead of the crack tip is increased significantly from that predicted by conventional elastic-plastic theory. Strain gradient plasticity theory is therefore useful for exploring size effects in solids at the microscale, and has recently attracted a great deal of interest. A stress formulation incorporating strain gradient effects has been derived in Fleck and Hutchinson ${ }^{24}$ as

$t_{k}=n_{i}\left(\sigma_{i k}-\frac{\partial \tau_{i j k}}{\partial x_{j}}\right)+n_{i} n_{j} \tau_{i j k}\left(D_{p} n_{p}\right)-D_{j}\left(n_{i} \tau_{i j k}\right)$

where $n_{i}$ are the components of the direction cosines on the considered plane. $D_{j}$ is a differential operator defined as $D_{j}=\partial / \partial x_{j}-n_{j} n_{k} \partial / \partial x_{k}$

Consider the plane along the $\mathrm{x}$-axis ahead of the crack tip, as shown in Fig. 1b. Then $n_{1}=0$ and $n_{2}=1$. One has

$t_{2}=\sigma_{22}-2 \frac{\partial \tau_{212}}{\partial x_{1}}-\frac{\partial \tau_{222}}{\partial x_{2}}=f\left(\frac{x}{R_{0}} ; \frac{E}{\sigma_{Y}}, v, \frac{T}{\sigma_{Y}}, N, \frac{L}{R_{0}}\right)$ where a length parameter $R_{0}$ is introduced and is defined as

$R_{0}=\frac{E \Gamma_{0}}{3 \pi\left(1-v^{2}\right) \sigma_{Y}^{2}}$

$R_{0}$ characterizes the plastic zone size in small scale yielding.

\section{A new finite element method for strain gradient problem}

Second order displacement-derivative terms are included in the constitutive equations including strain gradient effects, as shown in the Eqs (2) and (8). Thus, conventional finite element methods based on displacement continuity $\left(\mathrm{C}_{0}\right.$-continuity) are ineffective. Wei and Hutchinson $^{36}$ have developed a new finite element method by taking the pure displacement gradients as the node variables. They first considered a new form of expressing the variation equation

$\int_{V}\left\{\sigma_{i j} \delta U_{i j}+\tau_{i j k} \delta \frac{\partial U_{k j}}{\partial x_{i}}\right\} \mathrm{d} V=\int_{V} f_{i j}^{B} \delta U_{i j} \mathrm{~d} V+\int_{S} M_{j i} \delta U_{i j} \mathrm{~d} S$

where

$U_{i j}=\frac{\partial u_{i}}{\partial x_{j}}, f_{i j}^{B}=\sigma_{i j}-\tau_{k j i, k}, M_{j i}=n_{k} \tau_{k j i}$

From the Eq. (16) the fundamental variables are displacement gradients; displacements do not appear in the Eq. (16) directly. From the right hand side of the Eq. (16) an equivalent body force has been defined. Thus, for solving a problem, the first step is to choose the initial value of the equivalent body force. A complete formulation for a triangular element has been developed by Wei and Hutchinson. ${ }^{36}$ The node variables for each element are shown in Fig. 2c. The new finite element method has been tested by comparing the numerical results with the exact solutions obtained by Huang et al. ${ }^{33}$ In the present research, the adopted finite element mesh is shown in Fig. 2. In this, each quadrilateral or rectangular element is divided into four triangular elements, as shown in Fig. 2c. In the calculations, the centroid of each triangular element is taken as a unique Gauss point.

\section{CONSTRAINT EFFECTS ON THE STRESS DISTRIBUTION FOR A STATIONARY CRACK}

For the stationary crack case, we have adopted two kinds of finite element mesh in our analyses. One kind (Fig. 2a) is the conventional polar-coordinate mesh, i.e. keeping uniform division in the circular direction; the element size is increased simply with radial distance away from 
(a)

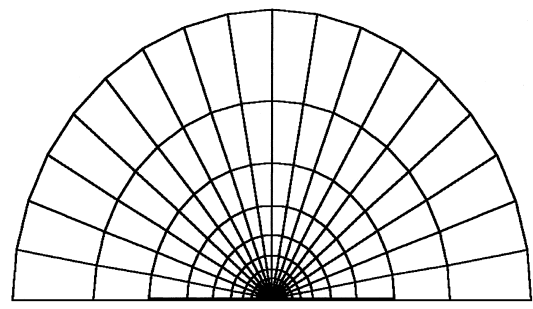

(b)

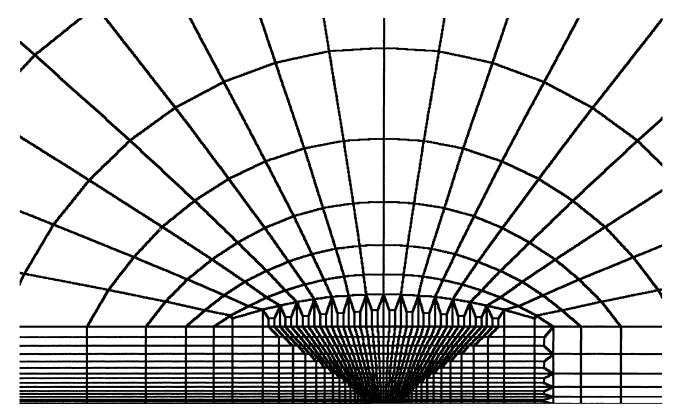

(c)

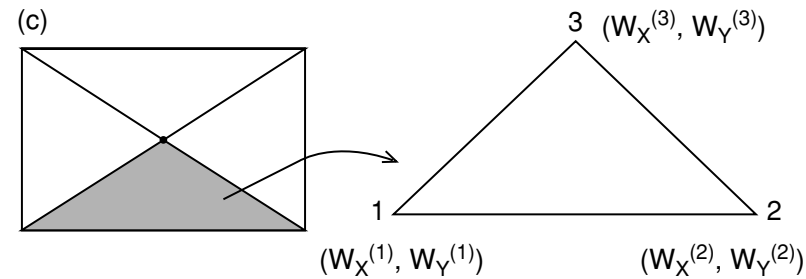

Fig. 2 Globe finite element mesh (a), near crack tip mesh (b) and a quadrilateral is divided into four triangular elements, displacement gradients are taken as the node variables (c).

the crack tip. The second form of mesh is more complicated. It is specially designed for the analysis of a steadily growing crack; it is shown in Fig. 2b. We found that the difference in the results from adopting either mesh type was small.

All the present calculations used the parameter values $E / \sigma_{Y}=300, v=0.3, N=0.2$. They compare the results for three different kinds of strain gradient effect; $L / R_{0}=0,0.2$ and 0.4 . The case $L / R_{0}=0$ is that of conventional elastic-plastic theory (no strain gradient effect). The normalized T-stress values should be taken within the region $-1<T / \sigma_{Y}<1$ to satisfy the small scale yielding condition.

Figure 3 shows the stress distribution along the plane ahead of the crack tip for $L / R_{0}=0.2$, and for several normalized T-stress values from -1.0 to 1.0 . The conventional elastic-plastic theory results (for $L / R_{0}=0$ ) are also shown in the figure. It is clear that both strain gradient effects and constraint effects are significant, especially for $x<0.03 R_{0}$. When the T-stress is positive (tensile stress), the constraint effect can be neglected.
However, when the T-stress is negative (compressive stress), there is a definite constraint effect that depresses the stress value within the strain gradient-dominated region. Figure 4 shows the stress distributions obtained by taking the normalized length parameter as $L / R_{0}=0.4$. Comparing the results in Figs 4 and 3, one sees that both strain gradient effects and constraint effects increase as $L / R_{0}$ increases; in that the strain gradient effects increase the stress values while the constraint effects decrease them. For comparison, the results using the conventional elastic-plastic theory are also shown in Fig. 4. It is obvious that, within the region $x<0.03 R_{0}$ and as crack tip is approached, the stress value increases rapidly owing to strain gradient effects.

\section{CONSTRAINT EFFECTS ON THE STRESS DISTRIBUTION FOR A STEADILY GROWING CRACK}

A specifically designed finite element mesh for a steadily growing crack is shown in Fig. 2b. Near the crack surface around the crack tip, the mesh contains an element 'strap' along the horizontal direction. Inside this strap, neighbouring elements in the horizontal direction will have the same $y$-coordinates for their Gauss points, so as to integrate Eq. (12) with respect to $x$ in the numerical calculation. Also within the strap, each quadrilateral is divided into four triangular elements, as shown in Fig. 2c, which has been formulated for a special finite element method by Wei and Hutchinson. ${ }^{36}$ When the quadrilateral is rectangular, the inner node inside the quadrilateral is located at the intersection point of the diagonal lines. When it is not rectangular, the inner node is chosen at the mid-point of the horizontal median line of the quadrilateral. The solution procedure follows the method of Dean and Hutchinson, a strategy used by other researchers (see Ref.[32]).

Figure 5 shows the stress distributions on the plane ahead of the crack tip for a length parameter $L / R_{0}=0.2$ and for several normalized T-stress values. The results using conventional elastic-plastic theory are also shown in the figure. Figure 5 shows, for the steadily growing crack, a very clear constraint effect when the T-stress is compressive (negative), with a strong decrease of the stress distribution within the strain gradientdominated region. The strain gradient effects are also clear. The results using the strain gradient theory gradually approach those of the conventional elastic-plastic theory as one moves away from the crack tip.

Figure 6 shows the results for a length parameter where $L / R_{0}=0.4$. The results in the Figs 6 and 5 , demonstrate that strain gradient effects increase considerably as $L / R_{0}$ increases from 0.2 to 0.4 . The constraint effects are also large. However, when the T-stress is tensile constraint 

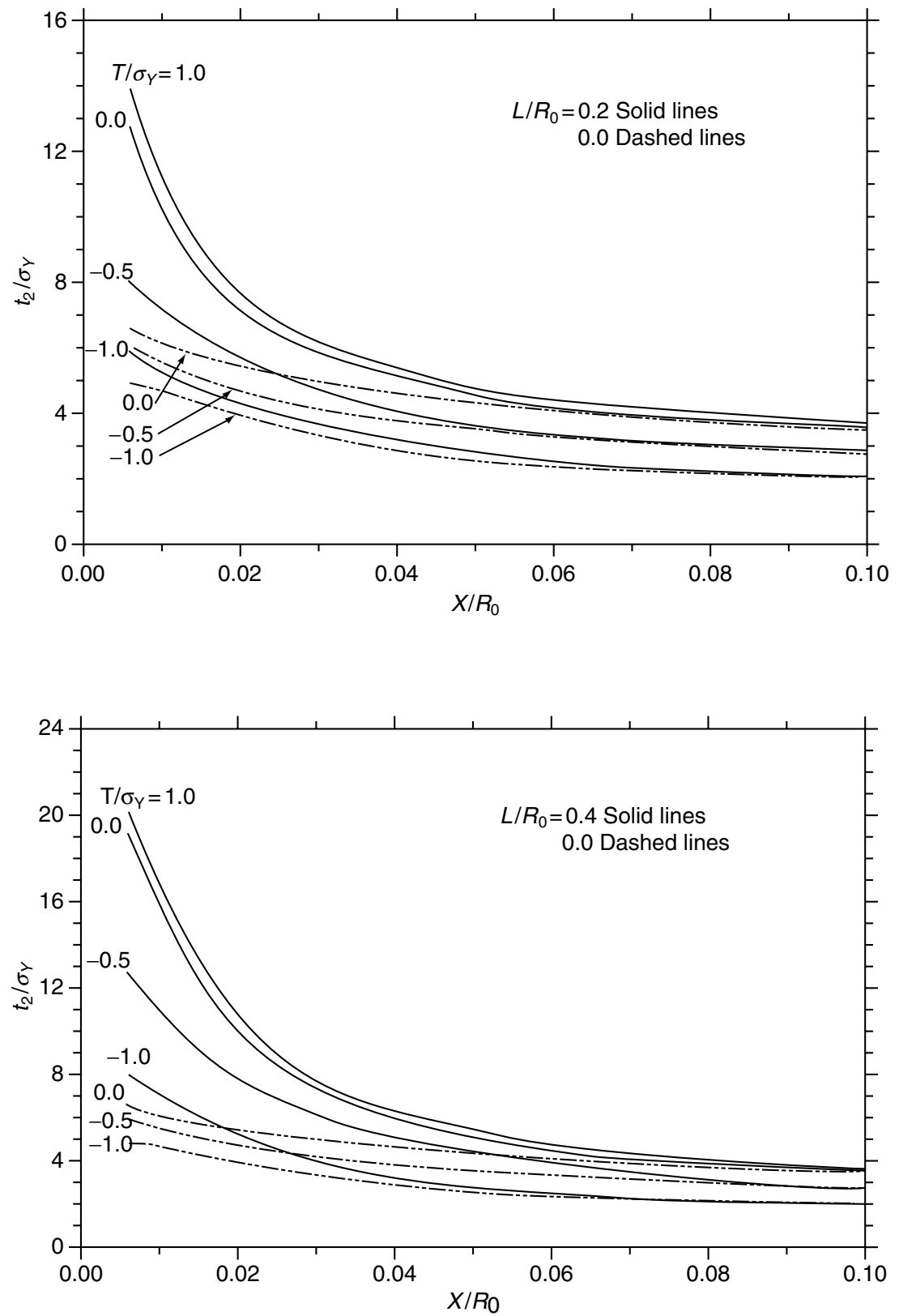

Fig. 3 Constraint effects on the stress distributions ahead of the crack tip in the strain gradient plasticity solid for the stationary crack case.
Fig. 4 Constraint effects on the stress distributions ahead of the crack tip in the strain gradient plasticity solid for the stationary crack case. effects are small and can be neglected. Comparing the results in Figs 5 and 6 with those in Figs 3 and 4, respectively, shows that the stress magnitude within the strain gradient-dominated region is lower for a growing crack than that for a stationary crack.

Figures 5 and 6 show that when a crack tip is steadily growing high values of the near separation stress are attainable; values of about 10 times the material yield stress (neglecting constraint effects). The corresponding value predicted by conventional elastic-plastic theory is comparatively small. Note that at distances further from the tip, the tractions drop slightly below those for the conventional solid, a fracture consistent with the requirement of overall force equilibrium that the higher tractions near tip be offset by lower values.

\section{THE INFLUENCE OF T-STRESS ON STEADY -} STATE FRACTURE TOUGHNESS

This section utilizes the SG (strain gradient) solid model with an EPZ model of Needleman ${ }^{13}$ and Tvergaard and Hutchinson. ${ }^{10}$ In this model, the traction-separation law characterizing the fracture process is prescribed as a boundary condition along the plane ahead of the crack tip, as depicted in Fig. 7. Attention here will focus on the steady-state and mode I plane strain toughness under 
Fig. 5 Constraint effects on the stress distributions ahead of the crack tip in the strain gradient plasticity solid for the steadily growing crack case.

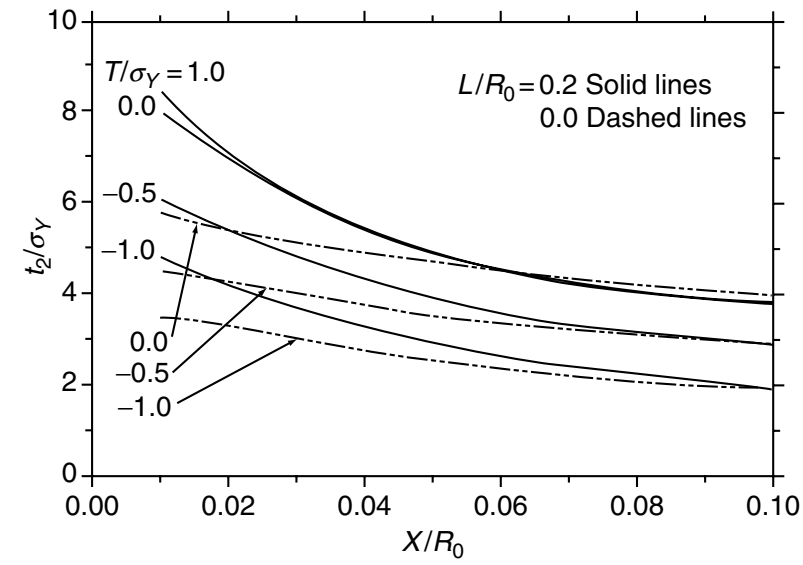

Fig. 6 Constraint effects on the stress distributions ahead of the crack tip in the strain gradient plasticity solid for the steadily growing crack case.

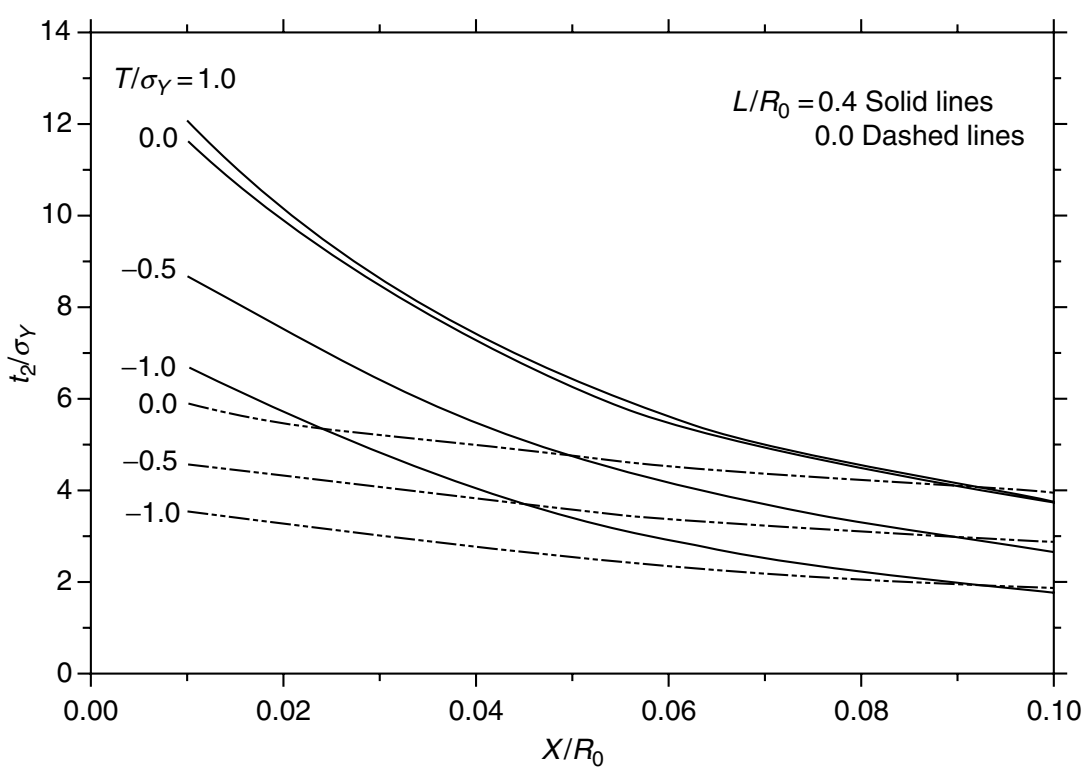

small scale yielding conditions. The results and discussion in the previous section show that strain gradient effects will substantially elevate the maximum separation strength $\hat{\sigma}$ of the EPZ model, while constraint effects (compressive T-stress) will considerably decrease it.

The form of the traction-separation law shown in Fig. 7 is the same as that employed in conventional theory (no strain gradient) (e.g.,Ref.[10]). However, within the context of the strain gradient theory, the traction on the crack surface is $t_{2}$ (see, Eq. (14)), not $\sigma_{22}$. Thus, in the present version of the EPZ model, the relation between $t_{2}$ and $\delta$ in Fig. 7 is prescribed as the condition along the plane ahead of the crack tip. The work of fracture per unit area, $\Gamma_{0}$, is related to $\hat{\sigma}$ and $\delta$ by

$\Gamma_{0}=\int_{0}^{\delta_{c}} t_{2} \mathrm{~d} \delta=\frac{1}{2} \hat{\sigma} \delta_{c}\left[1+\lambda_{2}-\lambda_{1}\right]$ where $\lambda_{1}=\delta_{1} / \delta_{c}$ and $\lambda_{2}=\delta 2 / \delta_{c}$. According to previous studies, the EPZ model results are insensitive to different values of $\lambda_{1}$ and $\lambda_{2}$ taken. In the present research, we take $\lambda_{1}=0.15, \lambda_{2}=0.5$ and $\delta_{c}$ as one-tenth of the minimum mesh size, as discussed by Tvergaard and Hutchinson. ${ }^{10-12}$ Thus from Eq. (18) the maximum separation strength $\hat{\sigma}$ will increase proportionally to $\Gamma_{0}$.

The numerical method discussed in section 2 applies to the steady-state problem. The iteration scheme must satisfy the traction-separation relation ahead of the tip and must adjust the level of the remote stress intensity factor $K$ and $T$ ( $K$ and $T$ increase proportionately) such that the steadily growing condition at the tip is met, i.e.

$\delta=\delta_{c}$ at $\quad x=0$

In the steady-state case, $K=K_{s s}$ and $T=T_{s s}$. The steady-state fracture toughness will be 

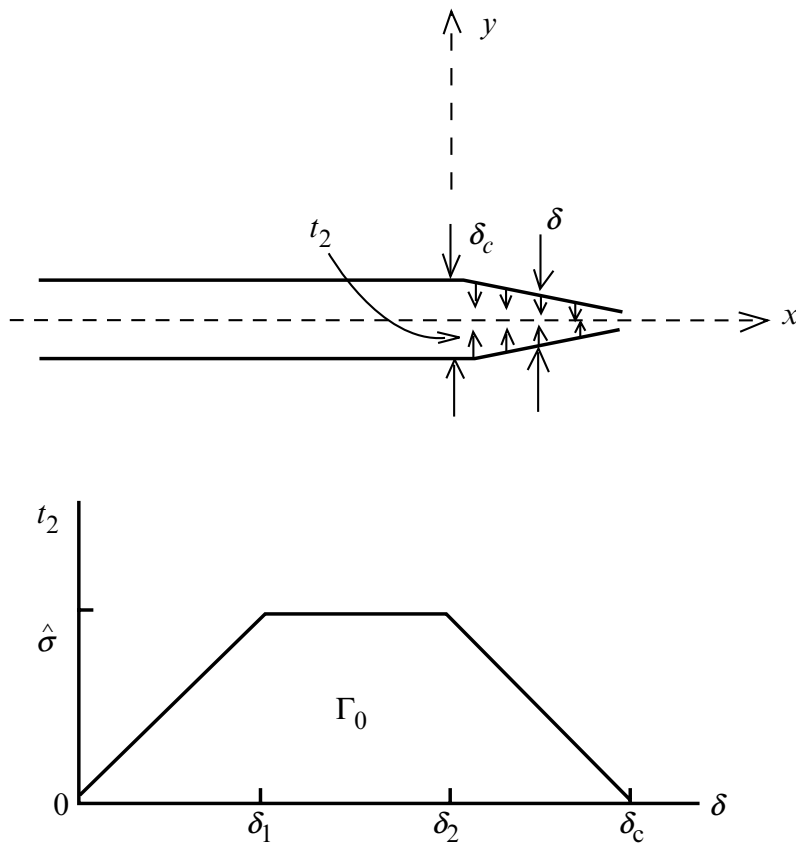

Fig. 7 Embedded process zone model (EPZ Model).

$\Gamma_{s s}=\frac{1-v^{2}}{E} K_{s s}^{2}$

which will depend on the T-stress effects indirectly through the T-stress influencing the crack tip elasticplastic field (see Ref.[12] for a detailed discussion). The $\Gamma_{s s}$ measures the total, or macroscopic, work of fracture; $\Gamma_{s s}-\Gamma_{0}$ is the plasticity contribution to the work of fracture. Dimensional considerations now give

$\frac{\Gamma_{s s}}{\Gamma_{0}}=F\left(\frac{E}{\sigma_{Y}}, v, N, \frac{\hat{\sigma}}{\sigma_{Y}}, \frac{L}{R_{0}}, \frac{T}{\sigma_{Y}}\right)$

In the analyses, our attention is focused on exploring the influences of T-stress, strain gradient effect and maximum separation strength on the steady-state fracture toughness. This is accomplished in all the calculations below, using the representative values of $E / \sigma_{Y}=300$, $v=0.3, N=0.2$.

Figure 8 shows the variation of normalized fracture toughness with normalized separation strength for several T-stress values. The results correspond to $L / R_{0}=0.2$. The fracture toughness increases with the maximum separation strength increases, especially for $\hat{\sigma} / \sigma_{Y}>4$ and, for this moderately high strain hardening $N=0.2$, fracture toughness increases sharply with increasing $\hat{\sigma} / \sigma_{Y}$. It is well known that for the elastic case $\Gamma_{s s} / \Gamma_{0}=1$. Clearly, the fracture toughness contributed from the plastic deformation is very big, specifically for $\hat{\sigma} / \sigma_{Y}>4$. On the other hand, it is obvious that during crack growth the maximum separation strength is large and the value exceeds six times the yield stress, i.e., $6<\hat{\sigma} / \sigma_{Y}<7.5$ for $L / R_{0}=0.2$, when T-stress effects are neglected. Considering T-stress effects (when the T-stress is compressive stress), one has $4<\hat{\sigma} / \sigma_{Y}<6$. These results are consistent with those shown in Fig. 5 within the strain gradient-dominated zone. Moreover, from Fig. 8, the constraint effects make the fracture toughness increase considerably.

Figure 9 shows the results for $L / R_{0}=0.4$, a case where the strain gradient effect is larger than that for $L / R_{0}=0.2$. Now, when the maximum separation strength is big, i.e., for $7<\hat{\sigma} / \sigma_{Y}<10$, the steady-state fracture toughness increases very rapidly as $\hat{\sigma} / \sigma_{Y}$ increases. The maximum separation strength for $L / R_{0}=0.4$ increases significantly compared with that for $L / R_{0}=0.2$ during steady crack growth. T-stress effects decrease the value of $\hat{\sigma} / \sigma_{Y}$. These results are also consistent with that in Fig. 6. Furthermore, from Fig. 9, T-stress effects make the fracture toughness increase remarkably.

\section{AN APPLICATION: INTERFACIAL FRACTURE TOUGHNESS OF GOLD/SAPPHIRE SYSTEM}

Lipkin et al. ${ }^{16}$ performed an experiment to measure the interfacial fracture toughness of the Gold/Sapphire system. The sample geometry, as shown in Fig. 1a, can be described as follows. Two thick ceramic (sapphire) materials are joined to a thin metal (gold) layer. There is a circular hole located at the sample centre. A pair of cracks on the symmetry plane near the hole boundary was created in advance of the experiment. These cracks will grow as compressive forces are exerted on the external boundaries. Lipkin et al. ${ }^{16}$ measured the steady-state fracture toughness for several different sample interfaces. Their experimental results can be summarized as the following four cases (from Fig. 13 in Ref.[16]):

1 For a carbon-treated sample, $\Gamma_{0} \approx 0.6 \mathrm{~J} / \mathrm{m}^{2}, \Gamma_{s s} \approx 2 \mathrm{~J} / \mathrm{m}^{2}$; 2 For a carbon-oxygen-treated sample, $\Gamma_{0} \approx 0.86 \mathrm{~J} / \mathrm{m}^{2}$, $\Gamma_{s s} \approx 230 \mathrm{~J} / \mathrm{m}^{2}$

3 For an as-bonded sample, $\Gamma_{0} \approx 0.9 \mathrm{~J} / \mathrm{m}^{2}, \Gamma_{s s} \approx 250 \mathrm{~J} / \mathrm{m}^{2}$;

4 For an oxygen-treated sample, $\Gamma_{0} \approx 0.9 \mathrm{~J} / \mathrm{m}^{2}, \Gamma_{s s} \approx 250$ $\mathrm{J} / \mathrm{m}^{2}$;

This data demonstrates that different interfacial fracture work $\Gamma_{0}$ and different steady-state fracture toughness $\Gamma_{s s}$ result from subjecting the interface of the metal/ceramic system to different treatment processes. The data can be strongly sensitive to the interface-treated conditions.

We shall apply our analysis of the last section to the prediction of this experiment. Firstly, consider some 
Fig. 8 The variations of the steady-state fracture toughness with peak separation strength.

Fig. 9 The variations of the steady-state fracture toughness with peak separation strength.
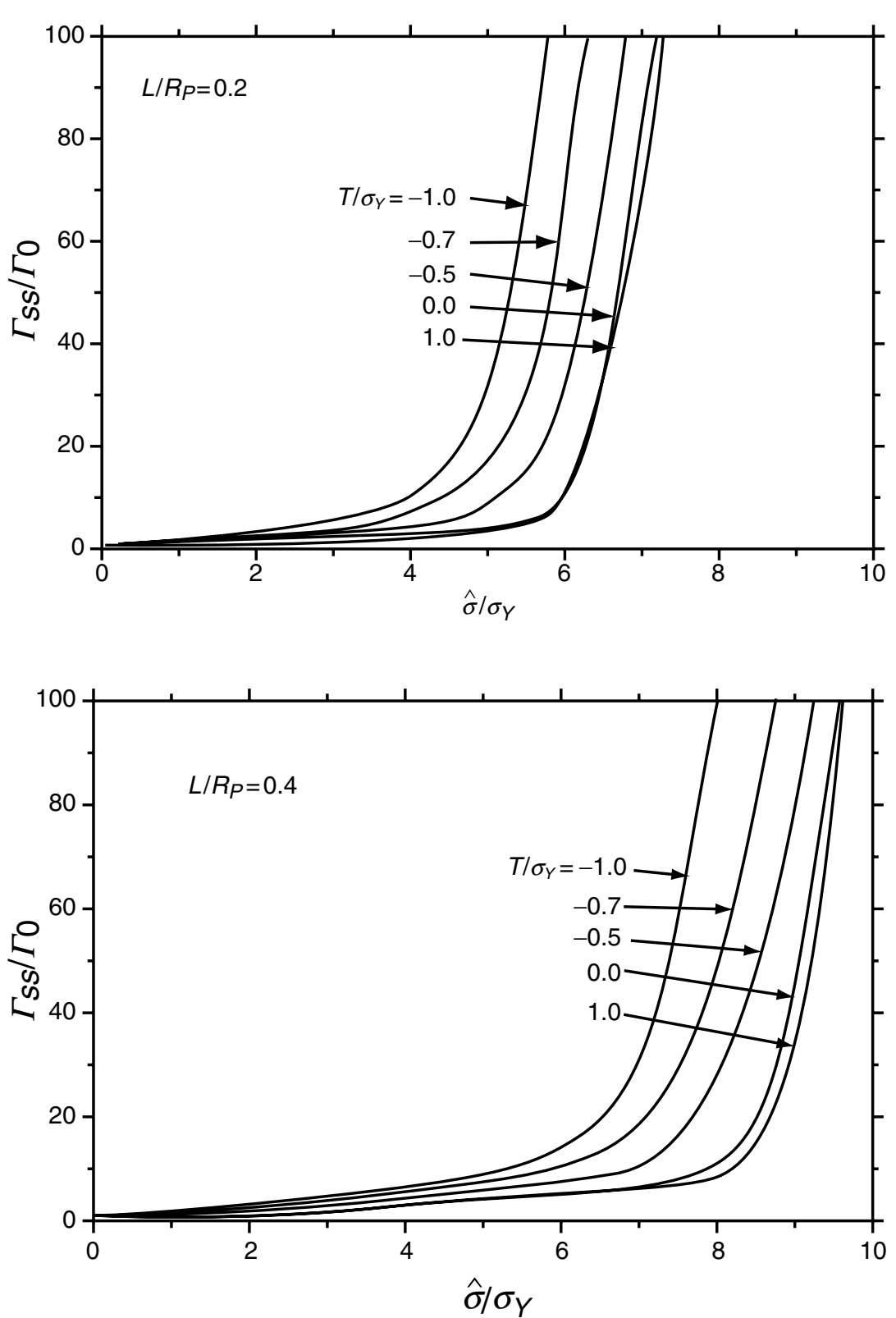

simplifications for the sample geometry, as shown in Fig. 1a. Taking an analytical cell around the crack tip, as shown in Fig. 1b, a K-field including the second term (T-stress) exerts on the boundary. This simplification is valid as long as the small scale yielding condition is satisfied. The magnitudes of $\mathrm{K}$ and $\mathrm{T}$ can be determined from the mechanical analysis of the sample for a stationary crack, or from the steady-state condition at the crack tip for a steadily growing crack. Secondly, neglect the influence of modulus mismatch on the fracture toughness, i.e., in the fracture toughness calculation, let $E_{s}=E_{m}=E$ and $v_{s}=v_{m}=v$. Under the above simplifications the results in the last section can be approximately applied to the experiment for measuring fracture toughness.
The sample interface treated with carbon (case 1) can be taken as a normal-strength interface, for which maximum separation strength should be predictable by conventional elastic-plastic theory. However, the other sample interfaces (cases 2-4) will correspond to higher-strength interfaces for which strain gradient plasticity theory could be used to predict their maximum separation strengths. For $L / R_{0}=0.2$, from the information in Fig. 5 predicts that, the maximum stress near the crack tip $t_{2}$ is about $4.5 \sigma_{Y}$ (for $T / \sigma_{Y}=-0.5$ ) for conventional elastic-plastic theory when the T-stress (compression) effects are considered. Thus, we take interface separation strength as $\hat{\sigma} \approx 4.5 \sigma_{Y}$ for case 1 and recall that the corresponding interfacial fracture work $\Gamma_{0}=2 \mathrm{Jm}^{-2}$. 
Using the proportional relation for $\Gamma_{0}$ and $\hat{\sigma}$ (see Eq. (18)), one will obtain the $\hat{\sigma}$ values for the other cases. The results are shown in Fig. 10. We have also investigated the results $L / R_{0}=0.4$ in Fig. 9 for predicting these experiments. However, a better fit, more consistent with the experiment, is from the case $L / R_{0}=0.2$.

Figure 10 shows a comparison of the numerical and experimental results. The numerical data are the same, as in Fig. 8, with a different coordinate scale. The simulation is basically consistent with the experiments. Furthermore, the length scale $L$ can be determined from a comparison of the simulation results with the experimental data. For the present simulation, we have taken $E / \sigma_{Y}=300$ and $v=0.3$. The data of Lipkin et al. ${ }^{16}$ for the Gold/Sapphire system, include the experimental values of $\Gamma_{0}=0.9 \mathrm{Jm}^{-2}$ and $\sigma_{Y}=10 \mathrm{MPa}$ therefore, one has $R_{0} \approx 2.7 \mu \mathrm{m}$ and $L \approx 0.54 \mu \mathrm{m}$ for this Gold/ Sapphire interface.

\section{CONCLUSIONS}

From a qualitative standpoint, the present analyses of the effects of constraint on elastic-plastic fracture behaviour in strain gradient solids capture the characteristics of fracture quite realistically. It shows that constraint plays an important role in the elastic-plastic fracture of strain gradient solids as it does for conventional elastic-plastic solids. Within the strain gradient-dominated region near the crack tip for either a stationary crack or a growing crack, the strain gradient effect makes the separation stress increase considerably, while the constraint effect (compressive T-stress) makes it recover to some extent. However, constraint effects cause the steady-state fracture toughness to increase considerably. In the previous studies using conventional elastic-plastic theory, the effects of T-stress (negative) also makes the separation stress decrease ${ }^{6}$ and cause the elevation of fracture toughness. ${ }^{12}$ Figures $3-6$, show that the strain gradient effect gradually diminishes, and the separation stress approaches result of conventional theory as one moves away from the crack tip.

The trends shown in Figs 8 and 9 are able to overcome the limitations of the EPZ model from conventional theory to a large extent. Specifically, with values of $L / R_{0}$ larger than about 0.4 , peak separation strengths well above $10 \sigma_{Y}$ can be attained. Atomic separation of a metal lattice or a metal/ceramic interface typically requires a work of separation, $\Gamma_{0}$, of the order of several $\mathrm{Jm}^{-2}$. Using representative values for $E$ and $\sigma_{Y}$ of metal materials, one finds values of $R_{0}$ around several micrometers, and the length scale $L$ in strain gradient theory is likely to be of the order of a micrometer. By contrast, when the fracture process is void nucleation, growth and coalescence ahead of the crack tip, the work of the fracture process, $\Gamma_{0}$, is approximately $1 \mathrm{KJm}^{-2}$, and $R_{0}$ is a fraction of a mm or more. ${ }^{10}$ In this case, $L / R_{0}$ is expected to be so small that strain gradient effects will have little influence on fracture toughness predictions. The constraint effects on void nucleation, growth and coalescence (the model of Gao et al. ${ }^{38}$ ) also suggest that the separation stress ahead of the crack tip must be quite low.

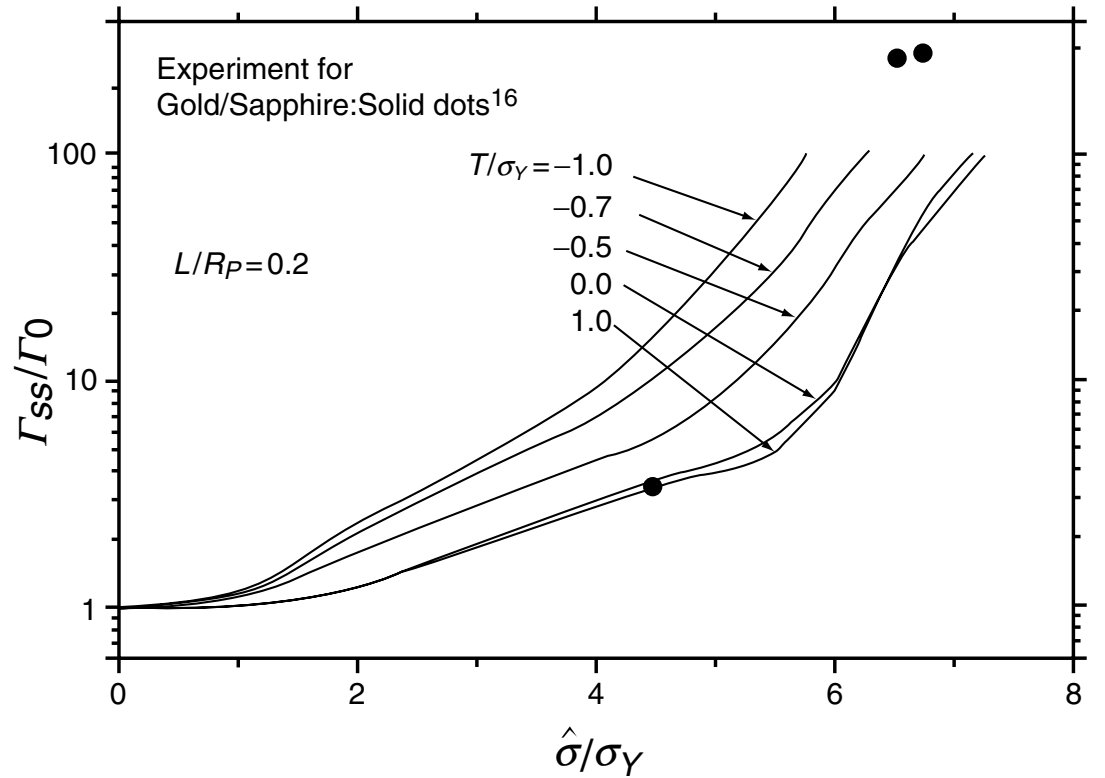

Fig. 10 The comparison of the simulation results with experimental results for the relations of the steady-state fracture toughness vs. peak separation strength. 


\section{APPENDIX}

Projection tensors of strain gradient components:

$$
\begin{aligned}
T_{i j k p q r}^{(1)}= & \frac{1}{6}\left\{\left(\delta_{i p} \delta_{j q}+\delta_{i q} \delta_{j p}\right) \delta_{k r}+\left(\delta_{j p} \delta_{k q}+\delta_{j q} \delta_{k p}\right) \delta_{i r}\right. \\
& \left.+\left(\delta_{i p} \delta_{k q}+\delta_{i q} \delta_{k p}\right) \delta_{j r}\right\} \\
& -\frac{1}{15}\left\{\left(\delta_{i j} \delta_{k r}+\delta_{j k} \delta_{i r}+\delta_{k i} \delta_{j r}\right) \delta_{p q}+\left(\delta_{i j} \delta_{k p}+\delta_{j k} \delta_{i p}\right.\right. \\
& \left.+\delta_{k i} \delta_{j p}\right) \delta_{q r} \\
& \left.+\left(\delta_{i j} \delta_{k q}+\delta_{j k} \delta_{i q}+\delta_{k i} \delta_{j q}\right) \delta_{r p}\right\}
\end{aligned}
$$

$$
\begin{aligned}
T_{i j k p q r}^{(2)}= & \frac{1}{12}\left\{e_{i k q} e_{j p r}+e_{j k q} e_{i p r}+e_{i k p} e_{j q r}+e_{j k p} e_{i q r}\right\} \\
& +\frac{1}{12}\left\{2\left(\delta_{i p} \delta_{j q}+\delta_{i q} \delta_{j p}\right) \delta_{k r}-\left(\delta_{j p} \delta_{k q}+\delta_{j q} \delta_{k p}\right) \delta_{i r}\right. \\
& \left.-\left(\delta_{k p} \delta_{i q}+\delta_{k q} \delta_{i p}\right) \delta_{j r}\right\}
\end{aligned}
$$

$$
\begin{aligned}
T_{i j k p q r}^{(3)}= & \frac{1}{12}\left\{-e_{i k q} e_{j p r}-e_{j k q} e_{i p r}-e_{i k p} e_{j q r}-e_{j k p} e_{i q r}\right\} \\
& -\frac{1}{8}\left\{\left(\delta_{i p} \delta_{j k}+\delta_{j p} \delta_{i k}\right) \delta_{q r}+\left(\delta_{i q} \delta_{j k}+\delta_{j q} \delta_{i k}\right) \delta_{p r}\right\} \\
& +\frac{1}{12}\left\{2\left(\delta_{i p} \delta_{j q}+\delta_{i q} \delta_{j p}\right) \delta_{k r}-\left(\delta_{j p} \delta_{k q}+\delta_{j q} \delta_{k p}\right) \delta_{i r}\right. \\
& \left.-\left(\delta_{k p} \delta_{i q}+\delta_{k q} \delta_{i p}\right) \delta_{j r}\right\} \\
& +\frac{1}{15}\left\{\left(\delta_{i j} \delta_{k r}+\delta_{j k} \delta_{i r}+\delta_{k i} \delta_{j r}\right) \delta_{p q}+\left(\delta_{i j} \delta_{k p}+\delta_{j k} \delta_{i p}\right.\right. \\
& \left.+\delta_{k i} \delta_{j p}\right) \delta_{q r} \\
& \left.+\left(\delta_{i j} \delta_{k q}+\delta_{j k} \delta_{i q}+\delta_{k i} \delta_{j q}\right) \delta_{r p}\right\}
\end{aligned}
$$

$$
T_{i j k p q r}^{(4)}=\frac{1}{8}\left\{\left(\delta_{i p} \delta_{j k}+\delta_{j p} \delta_{i k}\right) \delta_{q r}+\left(\delta_{i q} \delta_{j k}+\delta_{j q} \delta_{i k}\right) \delta_{p r}\right\}
$$

where $\delta_{i j}$ is Kronecker's delta. The $e_{i j k}$ is permutation tensor, i.e., $e_{i j k}=1,-1$ and 0 when $(i, j, k)$ are the cyclic permutations of $1,2,3$; other permutations of $1,2,3$ and equal for two of them, respectively.

\section{Acknowledgements}

The work was supported by National Science Foundations of China through Grants 19891180 and 19925211; and jointly supported by Fundamental Research Project from Chinese Academy of Sciences through Grant KJ951-1-201 and 'Bai Ren' Project. The work was also supported by Division of Engineering and Applied Sciences, Harvard University, and the NSF Grants CMS96-34632 in USA.

\section{REFERENCES}

1 Larsson, S. G. and Carlsson, A. J. (1973) Influence of nonsingular stress terms and specimen geometry on small-scale yielding at carck tips in elastic-plastic materials. F. Mech. Phys. Solids 21, 263-277.
2 Rice, J. R. (1974) Limitations to the small scale yielding approximation for crack tip plasticity. 7. Mech. Phys. Solids 22, $17-26$.

3 Li, Y. C. and Wang, T. C. (1986) Higher-order Asymptomatic solutions of the plane strain Mode I nonlinear crack. Scientia Sinica (Series A), 29, 941-955.

4 Betegon, C. and Hancock, J. W. (1991) Two-parameter characterization of elastic-plastic crack-tip fields. F. Appl. Mech. 113, 104-110.

5 Du, Z. Z. and Hancock, J. W. (1991) The effect of non-singular stresses on crack-tip constraint. F. Mech. Phys. Solids. 39, 555567.

6 O'Dowd, N. P. and Shih, C. F. (1991) Family of crack tip fields characterized by a triaxiality parameter-I. Structure of fields. 7. Mech. Phys. Solids 39, 989-1015.

7 O'Dowd, N. P. and Shih, C. F. (1992) Family of crack tip fields characterized by a triaxiality parameter-II. Fracture applications. 7. Mech. Phys. Solids 40, 939-963.

8 Wei, Y. and Wang, T. C. (1995) Fracture criterion based on the higher-order asymptotic fields. Int. F. Fracture. 73, 39-50.

9 Wei, Y. and Wang, T. C. (1995) Characterization of elasticplastic fields near stationary crack tip and fracture criterion. Engin. Fract. Mech. 51, 547-553.

10 Tvergaard, V. and Hutchinson, J. W. (1992) The relation between crack growth resistance and fracture process parameters in elastic-plastic solids. F. Mech. Phys. Solids. 40, $1377-1397$.

11 Tvergaard, V. and Hutchinson, J. W. (1993) The influence of plasticity on mixed mode interface toughness. 7. Mech. Phys. Solids. 41, 1119-1135.

12 Tvergaard, V. and Hutchinson, J. W. (1994) Effect of T-stress on mode I crack growth resistance in a ductile solid. Int. F. Solids Struct. 31, 823-833.

13 Needleman, A. (1987) A continuum model for void nucleation by inclusion debonding. 7. Appl. Mech. 54, 525-531.

14 Reimanis, I. E., Dalgleish, B. J. and Evans, A. G. (1991) The fracture resistance of a model metal/ceramic interface. Acta Metall. Mater. 39, 3133-3141.

15 Bagchi, A. and Evans, A. G. (1996) The mechanics and physics of thin film decohesion and its measurement. Interface Sci. 3, 169-193.

16 Lipkin, D. M., Clarke, D. R. and Evans, A. G. (1998) Effect of interfacial carbon on adhesion and toughness of gold-sapphire interface. Acta Mater. 46, 4835-4850.

17 Stelmashenko, N. A., Walls, M. G., Brown, L. M. and Milman, Y. V. (1993) Microindentations on W and Mo priented single crystals: An STM study. Acta Metall. Mater. 41, 2855-2865.

$18 \mathrm{Ma}$, Q. and Clarke, D. R. (1995) Size dependent hardness of solver single crystals. F. Mater. Res. 10, 853-863.

19 McElhaney, K. W., Vlassak, J. J. and Nix, W. D. (1998) Determination of indenter tip geometry and indentation contact area for depth-sensing indentation experiments. 7. Mater. Res. 13, 1300-1306.

20 Wei, Y., Wang, X., Wu, X. and Bai, Y. (2001) Theoretical and experimental researches of size effect in micro-indentation test. Science in Cbina (Series A), 44, 74-82.

21 Fleck, N. A., Muller, G. M., Ashby, M. F. and Hutchinson, J. W. (1994) Strain gradient plasticity: theory and experiments. Acta Metall. Mater. 42, 475-487. 
22 Stolken, J. S. and Evans, A. G. (1998) A microbend test method for measuring the plasticity length scale. Acta Mater: 46, 5109-5115.

23 Fleck, N. A. and Hutchinson, J. W. (1993) A phenomenological theory for strain gradient effects in plasticity. F. Mech. Phys. Solids. 41, 1825-1857.

24 Fleck, N. A. and Hutchinson, J. W. (1997) Strain gradient plasticity. Adv. Appl. Mech. 33, 295-361.

25 Aifantis, E. C. (1984) On the microstructural origin of certain inelastic models. Trans. ASME 7. Eng. Mater. Techn 106, 326-330.

26 Gao, H., Huang, Y., Nix, W. D. and Hutchinson, J. W. (1999) Mechanism-based strain gradient plasticity-I. Theory. F. Mech. Phys. Solids 47, 1239-1263.

27 Chen, S. and Wang, T. C. (2000) A new hardening law for strain gradient plasticity. Acta Mater. 48, 3997-4005.

28 Shu, J. Y. and Fleck, N. A. (1998) The prediction of a size effect in micro indentation. Int. F. Solids Struct. 35, 1363-1383.

29 Begley, M. R. and Hutchinson, J. W. (1998) The mechanics of size-dependent indentation. F. Mech. Phys. Solids. 46, 2049-2068.

30 Huang, Y., Gao, H., Nix, W. D. and Hutchinson, J. W. (2000) Mechnism-based strain gradient plasticity-II. Analysis. F. Mech. Phys. Solids 48, 99-128.
31 Xia, Z. C. and Hutchinson, J. W. (1996) Crack tip fields in strain gradient plasticity. 7. Mech. Phys. Solids 44, 1621-1648.

32 Wei, Y. and Hutchinson, J. W. (1997) Steady-state crack growth and work of fracture for solids characterized by strain gradient plasticity. 7. Mech. Phys. Solids. 45, 1253-1273.

33 Huang, Y., Chen, J. Y., Guo, T. F., Zhang, L. and Hwang, K. C. (1999) Analytic and numerical studies on. 35 mode I and mode II fracture in elastic-plastic materials with strain gradient effects. Int. F. Fract. 100, 1-27.

34 Wei, Y. (2000) Microscale mechanics for metal thin film delamination along ceramic substrates. Science in China (Series A), 43, 509-516.

35 Evans, A. G., Hutchinson, J. W. and Wei, Y. (1999) Interface adhesion: effects of plasticity and segregation. Acta Mater. 47, 4093-4113.

36 Wei, Y. and Hutchinson, J. W. (2001) A finite element method for strain gradient plasticity problem. Submitted for publication.

37 Dean, R. H. and Hutchinson, J. W. (1980) Quasi-static steady crack growth in small scale yielding. Fracture Mechanics, ASTM STP 700, 383-405.

38 Gao, X., Shih, C. F., Tvergaard, V. and Needleman, A. (1996) Constraint effects on the ductile-brittle transition in small scale yielding. F. Mech. Phys. Solids 44, 1255-1282. 